\title{
Chères lectrices, chers lecteurs,
}

Les considérations de type coût-utilité jouent un rôle central en médecine de nos jours. On attend de tous les protagonistes du système de santé qu'ils agissent de manière efficace, adéquate et économique. Dans ce contexte, l'arrêt rendu par le Tribunal fédéral au sujet d'une «défenestration» présente un intérêt certain. L'objet du jugement était le cas tragique d'un patient décédé pour s'être jeté dans le vide après une opération du cœur. De l'avis des juges fédéraux, une garde de nuit personnelle aurait dû être organisée dans ce cas d'espèce. Or, si une telle pratique s'établissait, elle entraînerait des coûts de l'ordre de 150 millions de francs par «année de vie humaine» sauvée. Un montant qui dépasse de beaucoup les coûts marginaux considérés comme raisonnables dans le débat général actuel sur les risques. Hanspeter Kuhn, responsable du service juridique de la FMH, commente l'affaire dans une perspective juridique $(\rightarrow$ page 1015$)$.

C'est également d'une pesée d'intérêts qu'il s'agit dans les directives de l'Académie suisse des sciences médicales (ASSM) concernant les biobanques. S'il convient de promouvoir la recherche en vue d'élargir le savoir (médical), il importe également de protéger les donneurs de matériel biologique, prélevé au bloc opératoire ou en laboratoire, contre une utilisation abusive de ces échantillons ou de données personnelles. En l'absence d'une législation spécifique au sujet des biobanques jusqu'à l'entrée en vigueur de la nouvelle loi relative à la recherche sur l'être humain, prévue pour 2010, ces nouvelles directives viennent combler une lacune et donneront une certaine sécurité tant aux donneurs qu'aux chercheurs ( $\rightarrow$ page 1021).

Comment prédire, au moyen d'un procédé aussi simple et d'une manière aussi précise que possible, les coûts générés dans un cabinet médical par tel ou tel collectif de patients? Les procédés qui se fondent principalement sur les indicateurs de santé que sont l'âge et le sexe, ne répondent que très partiellement à cette exigence, car ils ne tiennent pas compte des grandes fluctuations de coûts intervenant selon les individus au sein d'un groupe âge/sexe dé- terminé. Selon une étude utilisant les données de 46 cabinets de premier recours, l'indicateur de morbidité thurgovien (TMI), développé en vue d'optimiser les systèmes de rémunération dans le cadre de la gestion des soins (managed care), apporte à cet égard des améliorations substantielles pour le pronostic des coûts de santé individuels. $\rightarrow$ page 1038).

Organisé chaque année au Bürgenstock et accueillant de nombreux hôtes du monde de la politique et de la santé, le Séminaire des cadres de la Société suisse de médecine générale (SSMG) est un événement empreint de tradition, qui jouit d'un grand rayonnement. Les débats sont inspirés par le légendaire «esprit du Bürgenstock» qui, cette année, était particulièrement présent après la manifestation réussie des médecins de premier recours du $1^{\mathrm{er}}$ avril. Bruno Kesseli, rédacteur en chef du BMS, a profité de l'ambiance de renouveau qui régnait au séminaire pour tenter de suivre à la trace l'esprit du Bürgenstock... ( $\rightarrow$ page 1066).

La rédaction

\section{IMPRESSUM}

\section{Rédaction}

Dr et lic. phil. Bruno Kesseli

(Rédacteur en chef)

Dr Erhard Taverna

Rédaction Ethique

Christina Aus der $\mathrm{Au}$

Pr Lazare Benaroyo

Pr Christoph Rehmann-Sutter

Rédaction Histoire

PD Dr et lic. phil. Iris Ritzmann

Pr Jean Jacques Dreifuss

Dr Eberhard Wolff

Managing Editor

Thomas Heuer, lic. phil.

Secrétariat de rédaction

Margrit Neff

Adresse de la rédaction

EMH Editions médicales suisses SA

Farnsburgerstrasse 8, 4132 Muttenz

Case postale, 4010 Bâle

Tél. 06146785 55, fax 0614678556

E-mail: redaction.bms@emh.ch

Internet: www.bullmed.ch

\section{Délégués des sociétés de discipline} médicale

Allergologie et immunologie clinique: $\operatorname{Pr}$ A. Birche

Anesthésiologie: Pr P. Ravussin

Angiologie: Pr R. Koppensteiner

Cardiologie: Pr B. Meier

Chirurgie: $\operatorname{Pr}$ M. Decurtins

Chirurgie cardiaque et vasculaire thoracique:

Pr T. Carrel

Chirurgie de la main: PD Dr L. Nagy

Chirurgie maxillo-faciale: $\operatorname{Pr~H.-F.~Zeilhofer~}$

Chirurgie orthopédique: Dr T. Böni

Chirurgie orthopedique: Dr T. Bon

Chirurgie pédiatrique: Dr M. Bitte

Chirurgie plastique, reconstructive

et esthétique: Dr J. G. Poëll

Editeur

FMH

Fédération des médecins suisses

Verbindung der Schweizer Ärztinnen

und Ärzte

Federazione dei medici svizzeri

Swiss Medical Association

Elfenstrasse 18, 3000 Berne 15

Elfenstrasse 18, 3000 Berne 15
Tél. 03135911 11, fax 0313591112

E-mail: info@fmh.ch

Internet: www.fmh.ch

Editions

EMH Editores Medicorum Helveticorum

EMH Editions médicales suisses SA

EMH Schweizerischer Ärzteverlag AG

EMH Edizioni mediche svizzere SA

EMH Swiss Medical Publishers Ltd.

Case postale, 4010 Bâle

Tél. 06146785 55, fax 0614678556

E-mail: verlag@emh.ch

Internet: www.emh.ch

\section{Production}

Schwabe AG

Farnsburgerstrasse 8,4132 Mutten $z$

Tél. 0614678585 , fax 0614678586

E-mail: druckerei@schwabe.ch

Paraît le mercredi

Annonces

Pharmaceutiques

pharma media promotion

K. Hess

Lättichstrasse 6,6342 Baar 2

Tél. 04176023 23, fax 0417602327

E-mail:

info@pharma-media-promotion.ch

Non pharmaceutiques

Schwabe AG

Chantal Schneeberge

Frankfurtstrasse 14, Case postale 340

4008 Bâle

Tél. 06133311 07, fax 0613331106

E-mail: c.schneeberger@schwabe.ch

«Offres et demandes d'emploi»

EMH Editions médicales suisses SA

Farnsburgerstrasse 8, 4132 Muttenz

Tél. 0614678555 , fax 0614678556

E-mail: stellenmarkt@emh.ch

"Office de placement»

FMH Consulting Services

Office de placement

Case postale 246, 6208 Oberkirch

Tél. 04192500 77, fax 0419210586

E-mail: fmhstv@hin.ch

ISSN 1661-5948

Médecine nucléaire: Pr J. Müller

Médecine pharmaceutique: Dr P. Kleist

Médecine physique et réadaptation:

Dr M. Weber

Médecine tropicale et médecine de voyages:

PD Dr C. Hatz

Néonatologie: Pr H.-U. Bucher

Néphrologie: Pr J.-P. Guignard

Neurochirurgie: $\mathrm{Pr} \mathrm{H}$. Landolt

Neurologie: Pr H. Mattle

Neuropédiatrie: Pr J. Lütschg

Neuroradiologie: Pr W. Wichmann

Oncologie: PD Dr B. Pestalozzi

Ophtalmologie: Dr A. Franceschetti

Oto-rhino-laryngologie et chirurgie

cervico-faciale: Pr J.-P. Guyot
Marketing EMH

EMH Editions médicales suisses SA

Case postale, 4010 Bâle

Tél. 06146785 55, fax 0614678556

E-mail: marketing@emh.ch

\section{Abonnements}

Membres de la FMH

FMH Fédération des médecins suisses

Elfenstrasse 18, 3000 Berne 15

Tél. 03135911 11, fax 0313591112

Autres abonnements

EMH Editions médicales suisses SA

Abonnements, Case postale, 4010 Bâle

Tél. 0614678575 , fax 0614678576

E-mail: abo@emh.ch

Abonnement annuel: CHF 320-porto en plus

(C) 2006 by EMH Editions médicales suisses SA. Tous droits réservés. Toute reproduction d'article, y inclus électroniquement et la traduction, en totalité ou par extrait, est soumise à l'autorisation écrite des éditions.

ISSN 1424-4012 (édition électronique)

Pharmacologie et toxicologie clinique:

Dr T. Buclin

Pathologie: Pr G. Cathomas

Pédiatrie: Dr R. Tabin

Pneumologie: Pr E. Russi

Prévention et santé publique: $\operatorname{Dr} C$. Junker

Psychiatrie et psychothérapie: Dr W.J. Fuchs

Psychiatrie et psychothérapie d'enfants

et d'adolescents: Dr R. Hotz

Radiologie: Pr B. Marincek

Radio-oncologie: $\operatorname{Pr} \mathrm{R}$. Greiner

Rhumatologie: Pr M. Seitz

Urologie: PD Dr T. Zellweger
Médecine générale: $\mathrm{Dr}$ B. Kisslin

Médecine intensive: $\mathrm{Dr} C$. Jenn

Médecine légale: $\operatorname{Pr}$ T. Krompecher 